\title{
Effect of Fractional Fourier Transformation on Time-Frequency Distributions Belonging to the Cohen Class
}

\author{
Haldun M. Ozaktas, Nilgün Erkaya, and M. Alper Kutay
}

\begin{abstract}
We consider the Cohen class of time-frequency distributions, which can be obtained from the Wigner distribution by convolving it with a kernel characterizing that distribution. We show that the time-frequency distribution of the fractional Fourier transform of a function is a rotated version of the distribution of the original function, if the kernel is rotationally symmetric. Thus, the fractional Fourier transform corresponds to rotation of a relatively large class of time-frequency representations (phase-space representations), confirming the important role this transform plays in the study of such representations.
\end{abstract}

$\mathbf{T}$ THE fractional Fourier transform [1]-[3] has found many applications in quantum mechanics and optics [1], [2], [4]-[8], and signal processing [2], [3], [9]-[12], [23]. The fractional Fourier transform has been related to wavelet transforms [2], [13], neural networks [13], and is also related to various chirp-related operations [2], [14], [15]. It can be optically realized much like the usual Fourier transform [2], [4], [5], [7], and can be simulated with a fast digital algorithm [9], [16]. Other applications which are currently under study, or which have been suggested, include phase retrieval, signal detection, radar, tomography, and data compression.

The ath-order fractional Fourier transform $x_{a}(t)$ of the function $x(t)$ may be defined for $0<|a|<2$ as

$$
\begin{gathered}
x_{a}(t) \equiv \int_{-\infty}^{\infty} B_{a}\left(t, t^{\prime}\right) x\left(t^{\prime}\right) d t^{\prime} \\
B_{a}\left(t, t^{\prime}\right) \equiv \frac{\exp (-i \pi \operatorname{sgn}(\sin \phi) / 4+i \phi / 2)}{|\sin \phi|^{1 / 2}} \\
\cdot \exp \left[i \pi\left(t^{2} \cot \phi-2 t t^{\prime} \csc \phi+t^{\prime 2} \cot \phi\right)\right]
\end{gathered}
$$

where $\phi \equiv a \pi / 2$. The kernel approaches $B_{0}\left(t, t^{\prime}\right) \equiv \delta\left(t-t^{\prime}\right)$ and $B_{ \pm 2}\left(t, t^{\prime}\right) \equiv \delta\left(t+t^{\prime}\right)$ for $a=0$ and $a= \pm 2$, respectively.

The Wigner distribution $W_{x}(t, f)$ of a signal $x$ can be defined in terms of the time-domain representation of that signal $x(t)$ as [19], [20]

$$
W_{x}(t, f)=\int_{-\infty}^{\infty} x\left(t+t^{\prime} / 2\right) x^{*}\left(t-t^{\prime} / 2\right) e^{-2 \pi i f t^{\prime}} d t^{\prime} .
$$

Manuscript received February 23, 1995; approved June 7, 1995. The associate editor coordinating the review of this paper and approving it for publication was Dr. M. Unser.

The authors are with the Department of Electrical Engineering, Bilkent University, Bilkent, Ankara, Turkey.

Publisher Item Identifier S 1070-9908(96)01160-1.
Roughly speaking, $W_{x}(t, f)$ is a function which gives the distribution of signal energy over time and frequency. It is possible to show that the Wigner distribution of $x_{a}(t)$ is a rotated version of that of $x(t)$, a result independently arrived at by different authors [2], [5], [6], [3]. Let $\boldsymbol{R}_{\phi}$ denote the operator which rotates a $2-\mathrm{D}$ function counterclockwise by an angle $\phi$, that is

$$
R_{\phi}[z(t, f)]=z(t \cos \phi+f \sin \phi,-t \sin \phi+f \cos \phi) .
$$

Then, the above-mentioned result may be stated as

$$
W_{x_{a}}(t, f)=R_{-\phi}\left[W_{x}(t, f)\right] .
$$

A similar relation holds for the ambiguity function [2], [3]. A corollary of (4) is the following [2], [17]:

$$
\left\{\mathcal{R}_{\phi}\left[W_{x}(t, f)\right]\right\}\left(t_{a}\right)=\left|x_{a}\left(t_{a}\right)\right|^{2}
$$

where $\mathcal{R}_{\phi}$ is the Radon transform operator. $\mathcal{R}_{\phi}$ takes the integral projection of the 2-D function $W_{x}(t, f)$ onto an axis making angle $\phi=a \pi / 2$ with the $t$ axis. We refer to this axis as the $t_{a}$ axis, or the ath fractional Fourier domain. The concept of fractional Fourier domains is developed in [2] and [18].

In [3], Almeida further showed that a relation similar to (4) holds for (a modified form of) the short-time Fourier transform and spectrogram. This leads one to inquire whether a similar relation is valid for a more general class of time-frequency distributions. Here, we consider time-frequency distributions $T_{x}(t, f)$, which are members of the Cohen class. These distributions can be derived from the Wigner distribution through the relation [21], [22], [19]

$$
T_{x}(t, f)=\int_{t^{\prime}} \int_{f^{\prime}} \Psi_{T}\left(t-t^{\prime}, f-f^{\prime}\right) W_{x}\left(t^{\prime}, f^{\prime}\right) d t^{\prime} d f^{\prime} .
$$

$\Psi_{T}(t, f)$ is a kernel uniquely corresponding to the distribution $T_{x}$. We show below that $T_{x}$ will satisfy a relation similar to (4) if the kernel function is rotationally symmetric around the origin, that is, if $\Psi(t, f)$ is a function of $\left(t^{2}+f^{2}\right)^{1 / 2}$ only.

(The same condition can also be stated in terms of the alternative kernel function $\Phi(\theta, \tau)$, which is also employed in the study of the Cohen class [20], [19]. Since $\Phi(\theta, \tau)$ and $\Psi(t, f)$ constitute a 2-D Fourier transform pair, rotational symmetry of either implies rotational symmetry of the other.)

Thus, fractional Fourier transformation corresponds to rotation of not only the Wigner distribution, ambiguity function, and spectrogram, but a much larger class of time-frequency 
representations (phase-space representations). This not only confirms the important role this transform plays in the study of such representations, but also supports the notion of referring to the axis making angle $\phi=a \pi / 2$ with the $t$ axis as the $a$ th fractional Fourier domain [2], [18]. The many applications of the fractional Fourier transform have been predominantly posed in terms of the Wigner distribution of the signal. With this result, it will be possible to deal with other time-frequency distributions which might be more appropriate for particular applications.

Theorem: Let the signal $x$ have a time-frequency representation $T_{x}(t, f)$, which is a member of Cohen's class with kernel function $\Psi_{T}(t, f)$. Then,

$$
T_{\boldsymbol{x}_{a}}(t, f)=\mathbf{R}_{-\phi}\left[T_{x}(t, f)\right]
$$

for all $x$ and $\phi=a \pi / 2$, if $\Psi_{T}(t, f)$ is rotationally symmetric around the origin.

Proof: We only sketch the main features of the proof since the operations involved are elementary. First, apply $\boldsymbol{R}_{-\phi}$, as defined in (3), to both sides of (6) to obtain

$$
\begin{array}{r}
\boldsymbol{R}_{-\phi}\left[T_{x}(t, f)\right]=\int_{u} \int_{v} \Psi_{T}(t \cos \phi-f \sin \phi-u, t \sin \phi \\
+f \cos \phi-v) W_{x}(u, v) d u d v
\end{array}
$$

Now, consider an instance of (6) for the function $x_{a}(t)$, rather than $x(t)$. Use (4) and (3) to replace $W_{x_{a}}\left(t^{\prime}, f^{\prime}\right)$ by $W_{x}\left(t^{\prime} \cos \phi-f^{\prime} \sin \phi, t^{\prime} \sin \phi+f^{\prime} \cos \phi\right)$. Finally, make the change of variables $u=t^{\prime} \cos \phi-f^{\prime} \sin \phi, v=t^{\prime} \sin \phi+$ $f^{\prime} \cos \phi$, to obtain

$$
\begin{aligned}
T_{x_{a}}(t, f)=\int_{u} \int_{v} \Psi_{T}(t-u \cos \phi-v \sin \phi, f \\
+u \sin \phi-v \cos \phi) W_{x}(u, v) d u d v
\end{aligned}
$$

Equation (7) will be true if the right-hand sides of the last two equations are equal for all $x$ and for all $\phi$ (or $a$ ). This will be the case if

$$
\begin{aligned}
& \Psi_{T}(t \cos \phi-f \sin \phi-u, t \sin \phi+f \cos \phi-v) \\
& \quad=\Psi_{T}(t-u \cos \phi-v \sin \phi, f+u \sin \phi-v \cos \phi)
\end{aligned}
$$

for all of the appearing variables. Now, it is not difficult to show, by the transformation $t^{\prime}=t-u \cos \phi-v \sin \phi, f^{\prime}=$ $f+u \sin \phi-v \cos \phi$, that this condition is equivalent to $\Psi_{T}(t, f)$ being rotationally symmetric. This completes the proof.

\section{REFERENCES}

[1] A. C. McBride and F. H. Herr, "On Namias's fractional Fourier transform." IMA J. Appl. Math., vol. 39, pp. 159-175, 1987.

[2] H. M. Ozaktas, B. Barshan, D. Mendlovic, and L. Onural, "Convolution, filtering, and multiplexing in fractional Fourier domains and their relation to chirp and wavelet transforms," J. Opt. Soc. Amer. A, vol. 11 , pp. 547-559, 1994.

[3] . L. B. Almeida, "The fractional Fourier transform and time-frequency representations," IEEE Trans. Signal Processing, vol. 42, pp. 3084-3091, 1994.

[4] H. M. Ozaktas and D. Mendlovic, "Fourier transforms of fractional order and their optical interpretation," Opt. Commun., vol. 101, pp. 163-169, 1993.

[5] A. W. Lohmann, "Image rotation, Wigner rotation and the fractional Fourier transform,"J. Opt. Soc. Amer. A, vol. 10, pp. 2181-2186, 1993.

[6] D. Mendlovic, H. M. Ozaktas, and A. W. Lohmann, "Graded-index fibers, Wigner distribution functions, and the fractional Fourier transform," Appl. Opt., vol. 33, pp. 6188-6193, 1994.

[7] H. M. Ozaktas and D. Mendlovic, "Fractional Fourier optics," J. Opt. Soc. Amer. A, vol. 12, pp. 743-751, 1995.

[8] T. Alieva, V. Lopez, F. Aguillo-Lopez, and L. B. Almeida, "The fractional Fourier transform in optical propagation problems," J. Modern Opt., vol. 41, p. 1037, 1994.

[9] M. A. Kutay, H. M. Ozaktas, L. Onural, and O. Arikan, "Optimal filtering in fractional Fourier domains," in Proc. IEEE Int. Conf. Acoust. Speech, Signal Processing, Detroit, MI, 1995. Full paper submitted to IEEE Trans. Signal Processing.

[10] D. Mendlovic, H. M. Ozaktas, and A. W. Lohmann, "Fractional correlation," Appl. Opt., vol. 34, pp. 303-309, 1995.

[11] J. R. Fonollosa and C. L. Kikias, "A new positive time-frequency dis" tribution," in Proc. IEEE Int. Conf. Acoust., Speech, Signal Processing, 1994, pp. IV-301-IV-304.

[12] J. Wood and D. T. Barry, "Radon transformation of the Wigner spectrum," IEEE Trans. Signal Processing, vol. 42, pp. 3166-3177, 1994.

[13] S.-Y. Lee and H. H. Szu, "Fractional Fourier transforms, wavelet transforms, and adaptive neural networks," Opt. Eng., vol. 33, pp. 2326-2330, 1994.

[14] D. Mihovilovic and R. N. Bracewell, "Adaptive chirplet representation of signals on time-frequency plane," Electron. Lett., vol. 27, pp. 1159-1161, 1991.

[15] S. Mann and S. Haykin, "Chirplets and warblets: Novel time-frequency methods," Electron. Lett., vol. 28, pp. 114-116, 1992.

[16] H. M. Ozaktas, O. Arikan, M. A. Kutay, and G. Bozdaği, "Digital computation of the fractional Fourier transform," submitted to IEEE Trans. Signal Processing.

[17] A. W. Lohmann and B. H. Soffer, "Relationship between the Radon-Wigner and fractional Fourier transforms," J. Opt. Soc. Amer. A, vol. 11, pp. 1798-1801, 1994.

[18] H. M. Ozaktas and O. Aytür, "Fractional Fourier domains," Signal Processing, vol. 46, pp. 119-124, 1995.

[19] F. Hlawatsch and G. F. Bourdeaux-Bartels, "Linear and quadratic timefrequency signal representations," IEEE Signal Processing Mag., vol. 9, pp. 21-67, 1992.

[20] L. Cohen, "Time-frequency distributions-A review," Proc. IEEE, vol. 77, pp. 941-981, 1989.

[21] space formulation of quantum mechanics," J. Math. Phys., vol. 17, pp. $1863,1976$.

[22] _ "Generalized phase space distribution functions," J. Math. Phys., vol. 7 , p. 781, 1966.

[23] H. M. Ozaktas, B. Barshan, and D. Mendlovic, "Convolution and filtering in fractional Fourier domains," Opt. Rev., vol. 1, pp. 15-16, 1994. 